\title{
An Approximate Solution to Predator-prey Models Using The Differential Transform Method and Multi-step Differential Transform Method, in Comparison with Results of The Classical Runge-kutta Method
}

\author{
Adeniji A A ${ }^{1, *}$, Noufe H. A ${ }^{2}$, Mkolesia A $\mathbf{C}^{1}$, Shatalov M Y $\mathbf{Y}^{1}$ \\ ${ }^{1}$ Department of Mathematics and Statistics, Tshwane University of technology, Pretoria, South Africa \\ ${ }^{2}$ Department of Mathematics, King Abdulaziz University, Rabigh, Saudi Arabia
}

Received June 13, 2021; Revised August 26, 2021; Accepted September 21, 2021

Cite This Paper in the following Citation Styles

(a): [1] Adeniji A A, Noufe H. A, Mkolesia A C, Shatalov M Y, "An Approximate Solution to Predator-prey Models Using The Differential Transform Method and Multi-step Differential Transform Method, in Comparison with Results of The Classical Runge-kutta Method," Mathematics and Statistics, Vol.9, No.5, pp. 799-805, 2021. DOI: 10.13189/ms.2021.090520

(b): Adeniji A A, Noufe H. A, Mkolesia A C, Shatalov M Y, (2021). An Approximate Solution to Predator-prey Models Using The Differential Transform Method and Multi-step Differential Transform Method, in Comparison with Results of The Classical Runge-kutta Method. Mathematics and Statistics, 9(5), 799-805. DOI: 10.13189/ms.2021.090520

Copyright $@ 2021$ by authors, all rights reserved. Authors agree that this article remains permanently open access under the terms of the Creative Commons Attribution License 4.0 International License

\begin{abstract}
Predator-prey models are the building blocks of the ecosystems as biomasses are grown out of their resource masses. Different relationships exist between these models as different interacting species compete, metamorphosis occurs and migrate strategically aiming for resources to sustain their struggle to exist. To numerically investigate these assumptions, ordinary differential equations are formulated, and a variety of methods are used to obtain and compare approximate solutions against exact solutions, although most numerical methods often require heavy computations that are time-consuming. In this paper, the traditional differential transform (DTM) method is implemented to obtain a nu-merical approximate solution to prey-predator models. The solution obtained with DTM is convergent locally within a small domain. The multi-step differential transform method (MSDTM) is a technique that improves DTM in the sense that it increases its interval of convergence of the series expansion. One predator-one prey and two-predator-one prey models are considered with a quadratic term which signifies other food sources for its feeding. The result obtained numerically and graphically showspoint DTM diverges. The advantage of the new algorithm is that the obtained series solution converges for wide time regions and the solutions obtained from DTM and MSDTM are compared with so-lutions obtained using the classical Runge-Kutta method of order four. The results demonstrated is that MSDTM
\end{abstract}

computes fast, is reliable and gives good results compared to the solutions obtained using the classical Runge-Kutta method.

Keywords Differential Transformation Method, Multi-step Differential Transform Method, Runge-Kutta Method, System of ODE, Mathematica ${ }^{\circledR}$, Mathcad®, Maple ${ }^{\circledR}$.

\section{Introduction}

The purpose of this paper is to exclusively employ the approach of differential transform method (DTM), multi-step differential transformation method (MSDTM), and Runge-Kutta method (RKM to obtain numerical approximation to systems of first order differential equations exact solution can't be provided. This approach is a comparative analysis of predatorprey models that is re-modified and model real-life situations. As known that ODEs (linear and non-linear) are used to model realistic situation by researchers in different fields such as Biology (interaction between organism, bacteria and parasites[1], [2], [3]), Chemistry (chemical kinetics, rate of chemical reactions [4],[5]), Engineering (suspension systems, electric circuit, oscillations and vibrations [6], [7], [8]), Annular habitat ([9],[10]) and parameters estimation of predator- 
prey models([11],). A variety of methods by which we either obtain an exact or approximate solution are available by computation to obtain solution to systems of differential equations [12]. The DTM procedure is one of the many numerical methods for systems of ODEs [13],[14], which uses polynomial for approximation to obtain the exact solution representation that is differentiable. An extension of DTM is that the MSDTM procedure [15], [16], [17] is subdivided into intervals of equal step size, and while Runge-Kutta obtains numerical computation for comparative analysis.

\section{Differential Transformation Method}

The DTM technique uses the form of polynomial as the approximation to exact solution where the technique uses higher order Taylor series expansion method [18]. The differential transform method is an iterative procedure for obtaining Taylor series solution of differential equation [8], it's known that Taylor series method undergo heavy computational procedure for higher orders and this DTM technique can be used to obtain numerical and analytical solutions for linear and nonlinear ordinary differential equations for a small domain.

The DTM of a function $y_{i}(t)$ at point $t_{0}$ can be expressed as

$$
Y_{i}(k)=\frac{1}{k !} \cdot\left[\frac{d^{k}}{d t^{k}} y_{i}(t)\right]_{t=t_{0}},
$$

where $y_{i}(t)$ represents the original function, $Y_{i}(k)$ is the transformed function and $\frac{d^{k}}{d t^{k}}$ is the derivative with respect to $t$, such that $k=0,1,2,3, \ldots$.

By employing the differential inverse transform, $Y_{i}(k)$ is obtained as:

$$
y_{i}(t)=\sum_{k=0}^{\infty} Y_{i}(k)\left(t-t_{0}\right)^{k},
$$

for implementation purpose, $y_{i}(t)$ is expressed as an infinite series and represented as

$$
y_{i}(t) \approx \sum_{k=0}^{N} Y_{i}(k)\left(t-t_{0}\right)^{k},
$$

where $N$ is the convergence of natural values.

Combining both Equation (1) and Equation (2), we obtain:

$$
y_{i}(t)=\sum_{n=1}^{\infty} \frac{1}{k !}\left[\frac{d^{k}}{d t^{k}} y_{i}(t)\right]_{t=t_{0}}\left(t-t_{0}\right)^{k} .
$$

The following basic properties of DTM are studied according to [19],[20],[21],[22]. Properties of DTM as seen in Table 1:

The properties of the DTM are used to execute the DTM procedure in Mathematica. This implies that the differential transform method is derived from Taylor series expansion which locally converges to the exact solution or the approximate solution obtained from other numerical methods. By doing this, the error increases at some value iteratively during the procedure of execution with the algorithm. From the numerical values obtained in the Mathematica ${ }^{\circledR}$, it shows that DTM obtains accurate approximation at a small region around the initial point. To improve this outcome of the numerical approximation, its imperative to improve the method by employing the multi-step differential transform method.

\section{Multi-step differential transform method}

Despite the fact that DTM provides approximate solutions to nonlinear problems with respect to convergent series with approximate computational components. There exist some limitations and this is due to the convergence within a small region which results in to slow convergent rate. To overcome this limitation, the proposed multi-step differential transform method [23], [24],[18] in this section, we developed the MSDTM to obtain a better numerical approximation for the solutions to system of differential (nonlinear) equations but not to predator-prey systems (with logistic terms; one prey, one predator and two prey and one predator). This procedure details on the concept of intervalising the domain i.e dividing the interval into $M$ sub-intervals $\left[t_{m-1}, t_{m}\right] \ni m=1,2, \ldots, M$ of equal step size represented as $h=\frac{T}{M}$ which is equivalent to $t_{m}=m h$. To executes this, we apply the DTM to an arbitrary nonlinear ODE over an interval to obtain approximate solution represented as

$$
y_{i}(t)=\sum_{k=0}^{N} b_{1 k} t^{k} \quad: \quad t \in\left[0, t_{i}\right],
$$

and initial conditions is $y^{(k)}(0)$ = $d_{k}, \forall k=0,1,2, \ldots, p-1 . \quad$ The initial conditions $y_{m}^{(k)}\left(t_{m-1}\right)=y_{m-1}^{(k)}\left(t_{m-1}\right)$ will be used over each subinterval and the differential transform method will be applied to the arbitrary nonlinear ODE and replace $t_{0}$ by $t_{m-1}$ in Equation (1). The algorithm in Mathematica ${ }^{\circledR}$ repeats the process and generates a sequence of numerical approximate solution of $y_{m}(t)$, where $m=1,2, \ldots, M$ for the solution of $y(t)$, such that $y_{m}(t)=\sum_{k=0}^{N} Y_{m}(y)\left(t-t_{m-1}\right)^{k}, t \in\left[t_{m-1}, t_{m}\right]$.

\section{Classical Runge-Kutta Method}

The fourth-order Runge-Kutta method is implemented for obtaining approximate solutions of ODEs which are developed by mathematicians named duo C. Runge (1856-1927) and M.W Kutta (1867-1944), [25]. The Runge-Kutta method as a numerical method is used for comparative analysis to obtaining numerical approximation for the system of nonlinear differential equation [14] such as predator-prey model and applicable to economical models [26]. In this paper, the built-in function of the fourth order classical Runge-Kutta method is considered to obtain an approximate solution for an initial value problem (IVP) [27], to implement this method of solution, $x(t)$ and $y(t)$ represent the predator-prey population of the IVP over the time interval $t \in[a, b]$. The time interval is subdivided into $n$ equal 
Table 1. Basic properties of DTM

\begin{tabular}{|c|c|}
\hline Original Function & Transformed Function \\
\hline$y(t)=u(t) \pm v(t)$ & $Y(k)=U(k) \pm V(k)$, \\
\hline$y(t)=c u(t)$ & $Y(k)=c U(k)$, \\
\hline$y(t)=\frac{d^{n} u(t)}{d t^{n}}$ & $Y(k)=\frac{(k+n) !}{k !} \cdot U(k+n) \Rightarrow(k+1)(k+2) \ldots(k+n) U(k+n)$, \\
\hline$y(t)=u(t) v(t)$ & $Y(k)=\sum_{n=k_{1}}^{k} U\left(k_{1}\right) V\left(k-k_{1}\right)$, \\
\hline$y(t)=t^{n}$ & $Y(k)=\delta(k-n)$ when $\delta(k-n)=\left\{\begin{array}{cc|}1 & k=n \\
0 & k \neq n\end{array}\right.$, \\
\hline$y(t)=e^{\lambda t}$, & $Y(k)=\frac{\lambda^{k}}{k !}$. \\
\hline
\end{tabular}

intervals and the step size is represented as $h=\frac{(b-a)}{n}$ where $h$ denote the step size.

We consider a system of first order ODE,

$$
\begin{gathered}
x^{\prime}=f(t, x, y) \quad: \quad x\left(t_{0}\right)=y_{0}, \\
y^{\prime}=f(t, x, y) \quad: \quad y\left(t_{0}\right)=y_{0} .
\end{gathered}
$$

To obtain the solution through the built-in function of Mathematica ${ }^{\circledR} \quad 12.2 .0$, the algorithm is implemented for the numerical solution of $x(t)$ and $y(t)$.

\section{Methodology}

In this section, we consider a nonlinear system of ordinary differential equations, a competitive one prey, one predator model and two prey and one predator model. In implementing, differential transformation method, multi-step differential method and fourth order classical Runge-Kutta method, this methods will be investigated by an algorithm in Mathematica®and its built-in functions. The approximate solution of these methods would be obtained and compared by the built-in functions of Mathematica $\AA$, and Maple $\AA$. This procedure is employed to investigate the comparative analysis of the solution obtained approximately and method used in obtaining the solution.

\section{Formulation of predator-prey models}

An example of a predator-prey model is Lotka-Volterra equations [28] which is a pair of first order, nonlinear systems of differential equation and mostly used to model the dynamics of biological and chemical systems in which prey and predator is defined to interact within a habitat or community. Proposed independently by Alfred Lotka [29] and Vita Volterra [30], and later modeled as systems of ODE. In this paper, we consider a system with a quadratic term that defines the system to have other sources of income/feeding which affects their carrying capacity.

\subsection{One Predator and one Prey}

Consider the the competitive predator-prey model with a quadratic term that details other source of feeding

$$
\begin{array}{r}
x^{\prime}=a_{1} x-a_{2} x^{2}-a_{3} x y, \\
y^{\prime}=-a_{4} y+a_{5} x y, \\
x(0)=2, y(0)=1 .
\end{array}
$$

where $x(t)$ and $y(t)$ are the prey and predator population, and $a_{1}, a_{2}, a_{3}, a_{4}, a_{5}$ are non-negative coefficients.Biological interpretation of the parameters in the Table 2

For the model in Equation (8) and Equation (9), the solutions are computed using the DTM, MSDTM, and Runge-Kutta method. These computation values obtained are predicated using computer algebraic software: (Mathematica®12.2, Mathcad®and Maple () ). From the basic properties of the DTM, see Table 1, consider $a_{1}=a_{3}=a_{4}=a_{5}=1$ and $a_{2}=0.1$, then the transformation is obtained as

$$
\begin{aligned}
(k+1) X(k+1)= & X(k)-0.1 \sum_{k_{1}=0}^{k} X\left(k_{1}\right) X\left(k-k_{1}\right) \\
& -\sum_{k_{1}=0}^{k} X\left(k_{1}\right) Y\left(k-k_{1}\right), \\
(k+1) Y(k+1)= & -Y(k)+\sum_{k_{1}=0}^{k} X\left(k_{1}\right) Y\left(k-k_{1}\right) .
\end{aligned}
$$

Employing the differential inverse transform, the series expansion solution obtained in Equation (3) cab obtained as a representation in the series solution for predator-prey population

$$
\begin{aligned}
x(t)= & -0.400000000(t)-0.9200000000(t)^{2} \\
& +0.05066666667(t)^{3}+0.4065333333(t)^{4} \\
& +0.1021600000(t)^{5}-0.1434053334(t)^{6}, \\
y(t)= & +(t)+0.3000000000(t)^{2} \\
& -0.3400000000(t)^{3}-0.3323333333(t)^{4} \\
& -0.00302666668(t)^{5}+0.1611000000(t)^{6}
\end{aligned}
$$

Due to the numerical values, the first ten points are given while the graphical representation for each algorithm procedure is shown. From the graph of Figure 1, it shows the outcome of MSDTM and CRK4 are unidentifiable which validates MSDTM has one of many numerical methods that gives fast and effective numerical approximate solution to the One predator and one prey model among many other nonlinear differential equations as that of the result obtained using the classical Runge-Kutta model algorithm in Mathematica®12.2.

Comparative solution of one predator and one prey model in Table 3 implemented using the proposed methods.

\subsection{One Predator and Two Prey}

The model discussed describes the competitive activity between two prey and one predator population which is detailed 
An Approximate Solution to Predator-prey Models Using The Differential Transform Method and Multi-step Differential 802 Transform Method, in Comparison with Results of The Classical Runge-kutta Method

Table 2. Biological definition of parameters

\begin{tabular}{|c|c|}
\hline Parameters & Definition \\
\hline$a_{1}$ & Growth rate of the prey \\
\hline$a_{2}$ & Competition for other means of feeding between the prey and predator \\
\hline$a_{3}, a_{5}$ & Prey(predator) density reduces due to the interaction between the predator(prey) \\
\hline$a_{4}$ & Mortality rate of the predator \\
\hline
\end{tabular}

Table 3. Numerical approximation of DTM,MSDTM, Classical Runge-Kutta Method, \& Error analysis

\begin{tabular}{|l|l|l|l|l|l|l|}
\hline \multirow{2}{*}{ Dataset } & \multicolumn{2}{|c|}{ DTM } & \multicolumn{2}{c|}{ MSDTM/CRK4 } & \multicolumn{2}{c|}{-Error- } \\
& $x(t)$ & $y(t)$ & $x(t)$ & $y(t)$ & $x(t)$ & $y(t)$ \\
\hline 0 & 2 & 1 & 2 & 1 & & 0 \\
\hline 0.1 & 1.95089 & 1.10263 & 1.95089 & 1.10263 & 0 & 0 \\
\hline 0.2 & 1.88428 & 1.20876 & 1.88428 & 1.20876 & 0 & 0 \\
\hline 0.3 & 1.80198 & 1.31525 & 1.80199 & 1.31525 & $1.0 \times 10^{-5}$ & 0 \\
\hline 0.4 & 1.70675 & 1.41844 & 1.70678 & 1.41844 & $3.0 \times 10^{-5}$ & $2.0 \times 10^{-5}$ \\
\hline 0.5 & 1.60193 & 1.51445 & 1.60213 & 1.51447 & $2.0 \times 10^{-4}$ & $1.7 \times 10^{-4}$ \\
\hline 0.6 & 1.49088 & 1.5995 & 1.4919 & 1.59967 & $1.02 \times 10^{-3}$ & $9.0 \times 10^{-4}$ \\
\hline 0.7 & 1.37602 & 1.67005 & 1.37993 & 1.67095 & $3.91 \times 10^{-3}$ & $9.64 \times 10^{-3}$ \\
\hline 0.8 & 1.2573 & 1.72243 & 1.26972 & 1.72607 & $1.242 \times 10^{-2}$ & 3.642 \\
\hline 0.9 & 1.1301 & 1.75133 & 1.16415 & 1.76383 & $3.405 \times 10^{-2}$ & $1.25 \times 10^{-2}$ \\
\hline 1 & 0.98222 & 1.74661 & 1.06536 & 1.78408 & $3.14 \times 10^{-2}$ & $3.747 \times 10^{-2}$ \\
\hline
\end{tabular}

by Bazykin [31]. The following model will be considered based on the population of Buffalo, Lion, and hippopotamus density.

$$
\begin{array}{r}
x(t)=a_{1} x-a_{2} x^{2}-a_{3} x y+a_{4} x z, \\
y(t)=-b_{1} y+a_{5} x y-a_{6} y^{2}+b_{2} y z, \\
z(t)=b_{3} z+b_{4} x z-b_{5} y z-a_{7} z^{2}, \\
x(0)=2, y(0)=x(0)=1,
\end{array}
$$

where $x, y$, and $z$ are the Buffaloes, Lion and hippopotamus population, and the biological terms stated as $a_{1}, a_{2}, a_{3}, a_{4}, b_{1}, a_{5}, a_{6}, b_{2}, b_{3}, b_{4}, b_{5}$, and $a_{7}$. Biological definition of each term represented on the Equation (14) - Equation (16) are presented in Table 4:

The model in Equation (14) to Equation (16) have the following assumption:

- The coefficients that are non-negative are independent of time.

- The prey population is affected by other food source and not the predator alone.

- Predation is not taken into full consideration.

This section deals with the comparative analysis between DTM, MSDTM algorithm, and Classical Runge-Kutta method built-in function in Mathematica. The graphical representation shows the divergence in the DTM and (MSDTM, CRK4) and the first ten points were chosen among the numerical values generated with Mathematica $^{\circledR}$, Maple ${ }^{\circledR}$, and Mathcad ${ }^{\circledR}$, software are presented due to space for the purpose of this paper, this points obtained shows how the MSDTM gives a better result and less computation. Taking the biological term to be $a_{1}=a_{2}=0.1, a_{3}=0.2, a_{4}=0.5, a_{5}=0.05,=a_{6}=$ $0.2, a_{7}=0.1, b_{1}, b_{2}, b_{3}, b_{4}, b_{5}=1$, implementing the differen- tial transform method to obtain as:

$$
\begin{gathered}
(k+1) X(k+1)=a_{1} X(k)-a_{2} \sum_{k_{1}=0}^{k} X\left(k_{1}\right) X\left(k-k_{1}\right) \\
-a_{3} \sum_{k_{1}=0}^{k} X\left(k_{1}\right) Y\left(k-k_{1}\right)+a_{4} \sum_{k_{1}=0}^{k} X\left(k_{1}\right) Z\left(k-k_{1}\right), \\
(k+1) Y(k+1)=-b_{1} Y(k)+a_{5} \sum_{k_{1}=0}^{k} X\left(k_{1}\right) Y\left(k-k_{1}\right) \\
-a_{6} \sum_{k_{1}=0}^{k} Y\left(k_{1}\right) Y\left(k-k_{1}\right)+b_{2} \sum_{k_{1}=0}^{k} Y\left(k_{1}\right) Z\left(k-k_{1}\right), \\
(k+1) Z(k+1)=b_{3} Z(k)+b_{4} \sum_{k_{1}=0}^{k} X\left(k_{1}\right) z\left(k-k_{1}\right) \\
-b_{5} \sum_{k_{1}=0}^{k} Y\left(k_{1}\right) Z\left(k-k_{1}\right)-a_{7} \sum_{k_{1}=0}^{k} Z\left(k_{1}\right) Z\left(k-k_{1}\right) .
\end{gathered}
$$

Applying the differential inverse transform, we obtain the following series expansion solution for each population of $x(t), y(t)$, and $z(t)$ as

$$
\begin{aligned}
& x(t)=2+0.4000000000(t)+0.9700000000(t)^{2}+0.6473333336(t)^{3} \\
& +0.5862583335(t)^{4}+0.3778320001(t)^{5}+0.2555952808(t)^{6}
\end{aligned}
$$

$$
y(t)=1-0.1000000000(t)+0.9750000000(t)^{2}+0.5073333333(t)^{3}
$$$$
+0.7402458335(t)^{4}+0.6275393334(t)^{5}+0.6047169736(t)^{6} \text {, }
$$

$$
z(t)=1+1.900000000(t)+1.960000000(t)^{2}+1.370666667(t)^{3}
$$$$
+0.7082250002(t)^{4}+0.2314674999(t)^{5}-0.05173495809(t)^{6} \text {. }
$$ 
Table 4. Biological definition of parameters

\begin{tabular}{|c|c|}
\hline Parameters & Definition \\
\hline$a_{1}, b_{3}$ & Prey population growth \\
\hline$b_{1}$ & Predator population growth \\
\hline$a_{2}, a_{6}, a_{7}$ & The prey are affected by other food sources by the predator \\
\hline$a_{3}, a_{4}, a_{5}, b_{2}, b_{4}, b_{5}$ & Interaction between the two preys and one predator \\
\hline
\end{tabular}

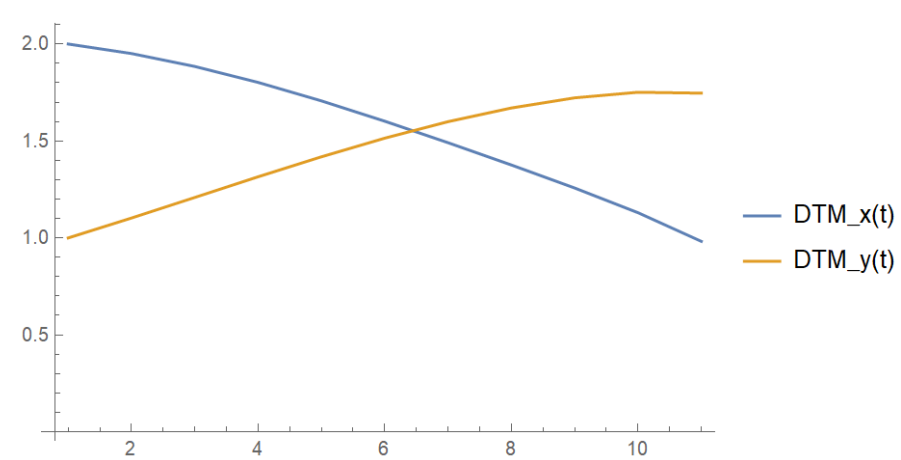

(a)

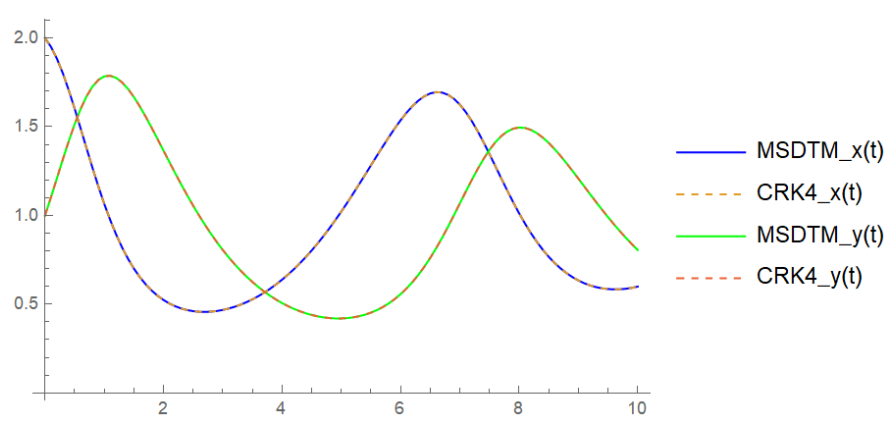

(b)

Figure 1. Graphical representation of $x(t), y(t)$ :(a) DTM plot and (b) MSDTM and CRK4

Graphical representation in Figure 2 shows the numerical solution of the method. It depicts the differential transform method doesn't give a good result as it's seen numerically from Table 5, how at some point of $t$, DTM begins to diverge which led to the dysfunctional graph obtained. It's imperative to know from the graph that DTM converges locally within the range of its initial point. To validate this for the benefit of this paper, only the first ten points were taken to show numerically the comparative analysis of the approximate solution of the methods.

The Table 5 shows numerically the approximate solution comparatively two-one prey-predator model.

\section{Discussion and Conclusion}

In this work, we carefully propose a multi-step differential transform method against the differential transform method and classical Runge-Kutta, which is a reliable modification of DTM that improves the convergence of the series expansion solution. This method provides immediate computation and less energy consumption to obtain numerical approximate solution to the prey-predator models that do not have an exact solution. Applying the multi-step DTM to the prey-predator

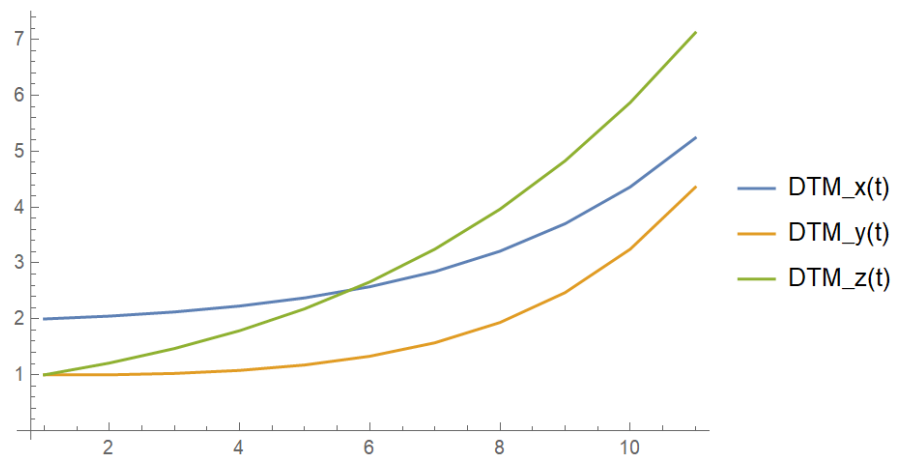

(a)

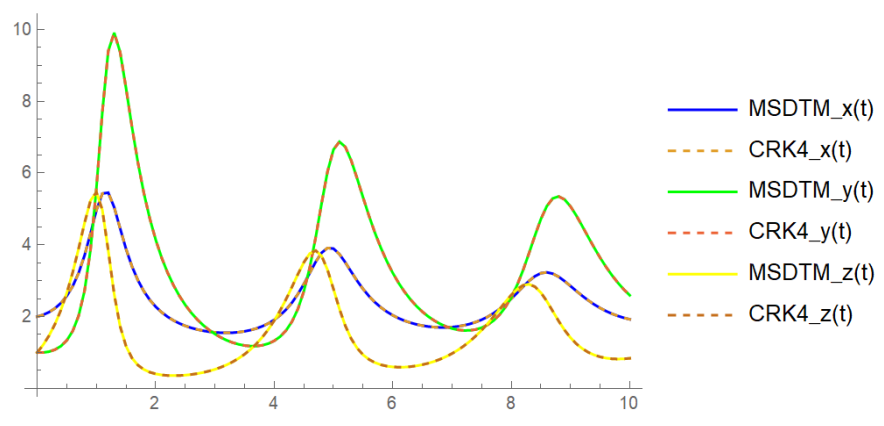

(b)

Figure 2. Graphical representation of $x(t), y(t), z(t)$ : (a) DTM plot (b) MSDTM and CRK4

models of samples $1 \& 2$, it can be observed that MSDTM gives a better approximation result against the CRK4 or the same results. From the graph of Figure 1 and Figure 2 seen, the images are unidentifiable which can be seen as well in the numerical values in the Table 3 and Table 5. From both tables Table 3 and Table 5, it shows that MSDTM gives an accurate approximate solution in comparison to CRK4, the absolute error for MSDTM against CRK4 was not presented because graphically its unidentifiable and numerically the same. From Table 3, it can be deduced that the values of DTM against MSDTM or CRK4, at a small domain it approximate the solution well but diverges as the domain becomes large.

The DTM, which result is predicated on the Taylor series expansion, the numerical values as well as graphical representation shows it diverges in the large domain due to its local converges in a small domain. Therefore the proposed method is efficient and an accurate method that provides analytical solutions to non-linear systems of differential equations in biology and chemistry. Figure 1 and Figure 2 of systems of ODEs show the solution obtained using the DTM has a small interval of convergence while the solution obtained using MSDTM which is compared with CRK4 has wide of intervals of convergence. 
An Approximate Solution to Predator-prey Models Using The Differential Transform Method and Multi-step Differential 804

Transform Method, in Comparison with Results of The Classical Runge-kutta Method

Table 5. Numerical approximation of DTM,MSDTM,\& Classical Runge-Kutta Method

\begin{tabular}{|l|l|l|l|l|l|l|l|l|l|l|}
\hline & \multicolumn{3}{|c|}{ DTM } & \multicolumn{3}{|c|}{ MSDTM/CRK4 } & \multicolumn{3}{|c|}{ Error } \\
& $x(t)$ & $y(t)$ & $z(t)$ & $x(t)$ & $y(t)$ & $z(t)$ & $x(t)$ & $y(t)$ & $z(t)$ \\
\hline 0 & 2 & 1 & 1 & 2 & 1 & 1 & & 0 & 0 \\
\hline 0.1 & 2.05041 & 1.00034 & 1.21104 & 2.05041 & 1.00034 & 1.21104 & 0 & & 0 \\
\hline 0.2 & 2.12505 & 1.02448 & 1.47057 & 2.12506 & 1.02449 & 1.47057 & $1.0 \times 10^{-5}$ & $1.0 \times 10^{-5}$ & 0 \\
\hline 0.3 & 2.23063 & 1.07941 & 1.78967 & 2.23066 & 1.07956 & 1.7896 & $3.0 \times 10^{-5}$ & $1.5 \times 10^{-5}$ & $7.0 \times 10^{-5}$ \\
\hline 0.4 & 2.37655 & 1.17632 & 2.18161 & 2.37678 & 1.17754 & 2.18095 & $2.3 \times 10^{-4}$ & $5.99 \times 10^{-1}$ & $6.6 \times 10^{-4}$ \\
\hline 0.5 & 2.57586 & 1.33249 & 2.66202 & 2.5769 & 1.33902 & 2.65814 & $1.04 \times 10^{-3}$ & $6.53 \times 10^{-3}$ & $3.88 \times 10^{-3}$ \\
\hline 0.6 & 2.84631 & 1.57353 & 3.24904 & 2.84959 & 1.59992 & 3.23161 & $3.28 \times 10^{-3}$ & $2.639 \times 10^{-2}$ & $1.743 \times 10^{-2}$ \\
\hline 0.7 & 3.21167 & 1.93611 & 3.9634 & 3.21855 & 2.02381 & 3.89791 & $6.88 \times 10^{-3}$ & $8.77 \times 10^{-2}$ & $6.5 \times 10^{-2}$ \\
\hline 0.8 & 3.70318 & 2.47111 & 4.82856 & 3.70727 & 2.72068 & 4.61256 & $4.09 \times 10^{-3}$ & $2.49 \times 10^{-1}$ & $2.16 \times 10^{-1}$ \\
\hline 0.9 & 4.36119 & 3.2472 & 5.87067 & 4.31639 & 3.85783 & 5.23432 & $4.48 \times 10^{-2}$ & $6.1 \times 10^{-1}$ & $6.3 \times 10^{-1}$ \\
\hline 1 & 5.23702 & 4.35484 & 7.11862 & 4.96545 & 5.58343 & 5.46656 & $8.8 \times 10^{-1}$ & $1.2 \times 10^{0}$ & $1.65 \times 10^{0}$ \\
\hline
\end{tabular}

This validates the algorithm proposed increases the interval of convergence for the series solution and the accuracy of the proposed algorithm can be accurate if the $h$ becomes smaller and sub-interval $k$ becomes larger. The proposed method MSDTM can be seen to be a reliable, accurate, and fast method compared with the classical RK4 built-in function in Mathematica ${ }^{\circledR}$ for a one prey one predator, and two prey one predator model with a quadratic term which signifies other sources of feeding. It can be concluded that MSDTM can be applied to other nonlinear systems of differential equations to obtain the same or better results with other existing methods.

\section{Acknowledgements}

The authors wish to thank Tshwane University of Technology for their financial support and the Department of Higher Education and Training, South Africa.

\section{REFERENCES}

[1] Abolfazl Tari. The differential transform method for solving the model describing biological species living together. Iranian journal of mathematical sciences and informatics (IJMSI), pages 63-74, 2012.

[2] Abdul-Monim Batiha and Belal Batiha. Differential transformation method for a reliable treatment of the nonlinear biochemical reaction model. Advanced studies in Biology, 3(8):355-360, 2011.

[3] A Harir, S Melliani, H El Harfi, and LS Chadli. Variational iteration method and differential transformation method for solving the seir epidemic model. International Journal of Differential Equations, 2020, 2020.

[4] Caihong Fan, Ping Li, and Yingzhen Xue. Application of differential equations in enzyme kinetics. Chemical Engineering Transactions, 71:883-888, 2018.

[5] Meksianis Z Ndii, Nursanti Anggriani, and Asep K Supriatna. Application of differential transformation method for solving dengue transmission mathematical model. In AIP conference proceedings. AIP Publishing LLC, 2018.
[6] JK Zhou. Differential transformation and its applications for electrical circuits, 1986.

[7] Shih-Shin Chen et al. Application of the differential transformation method to the free vibrations of strongly non-linear oscillators. Nonlinear analysis: Real world applications, 10(2):881-888, 2009.

[8] AS Oke. Convergence of differential transform method for ordinary differential equations. Journal of Advances in Mathematics and Computer Science, pages 1-17, 2017.

[9] Adejimi A Adeniji, I Fedotov, Michael Y Shatalov, and ACPG Mkolesia. Numerical investigation of diffusive predator-prey model with application to annular habitat. Biomath Communications Supplement, 5(1), 2018.

[10] AA Adeniji, MY Shatalov, I Fedotov, and AC Mkolesia. Inverse problems of the holling-tanner model with incomplete information. Discontinuity, Nonlinearity, and Complexity, 2021.

[11] Adejimi Adesola Adeniji, Igor Fedotov, and Michael Y Shatalov. Inverse problem of the holling-tanner model and its solution. BIOMATH, 7(2):1812057, 2018.

[12] Yalçın Öztürk. Numerical solution of systems of differential equations using operational matrix method with chebyshev polynomials. Journal of Taibah University for Science, 12(2):155-162, 2018.

[13] Minakshi Mohanty and Saumya R Jena. Differential transformation method (dtm) for approximate solution of ordinary differential equation (ode). Journal homepage: http://iieta. org/Journals/AMA/AMA_B, 61(3):135-138, 2018.

[14] Martha LL Abell and James P Braselton. Differential equations with Mathematica. Academic Press, 2016.

[15] Noufe H Aljahdaly. New application through multistage differential transform method. In AIP Conference Proceedings. AIP Publishing LLC, 2020. 
[16] Pakwan Riyapan, Sherif Eneye Shuaib, Arthit Intarasit, and Khanchit Chuarkha. Applications of the differential transformation method and multi-step differential transformation method to solve a rotavirus epidemic model. Mathematics and Statistics, 9(1), 2021. https://doi.org/10.13189/ms.2021.090112.

[17] Brahim Benhammouda and Hector Vazquez-Leal. A new multi-step technique with differential transform method for analytical solution of some nonlinear variable delay differential equations. SpringerPlus, 5(1):1-17, 2016.

[18] JMW Munganga, JN Mwambakana, Riette Maritz, TA Batubenge, and GM Moremedi. Introduction of the differential transform method to solve differential equations at undergraduate level. International Journal of Mathematical education in Science and technology, 45(5):781-794, 2014.

[19] F Karakoç and H Bereketoğlu. Solutions of delay differential equations by using differential transform method. International Journal of Computer Mathematics, 86(5):914-923, 2009.

[20] Aytac Arikoglu and Ibrahim Ozkol. Solution of differential-difference equations by using differential transform method. Applied Mathematics and Computation, 181(1):153-162, 2006.

[21] Ming-Jyi Jang, Chieh-Li Chen, and Yung-Chin Liy. On solving the initial-value problems using the differential transformation method. Applied Mathematics and Computation, 115(2-3):145-160, 2000.

[22] IH Abdel-Halim Hassan. Differential transformation technique for solving higher-order initial value problems. Applied Mathematics and Computation, 154(2):299-311, 2004.
[23] Noufe H Aljahdaly and SA El-Tantawy. On the multistage differential transformation method for analyzing damping duffing oscillator and its applications to plasma physics. Mathematics, 9(4):432, 2021.

[24] Ismail Tabet, Mohamed Kezzar, Meriem Chieul, Nouredin Nafir, and Abelkader Khentout. Application of multi-step differential transformation method (ms$\mathrm{dtm}$ ) for solving nonlinear problem of heat transfer in solar air collector. Journal homepage: http://iieta. org/journals/ijht, 37(2):481-486, 2019.

[25] Delin Tan and Zheng Chen. On a general formula of fourth order runge-kutta method. Journal of Mathematical Science \& Mathematics Education, 7(2):1-10, 2012.

[26] Giovanni Romeo. Elements of Numerical Mathematical Economics with Excel: Static and Dynamic Optimization. Academic Press, 2019.

[27] E Juarlin. Solution of simple prey-predator model by runge kutta method. In Journal of Physics: Conference Series. IOP Publishing, 2019.

[28] Nicolas Bacaër. Lotka, volterra and the predator-prey system (1920-1926). In A short history of mathematical population dynamics, pages 71-76. Springer, 2011.

[29] Alfred James Lotka. Elements of physical biology. Williams \& Wilkins, 1925.

[30] Vito Volterra. Fluctuations in the abundance of a species considered mathematically 1, 1926.

[31] Alexander D Bazykin. Nonlinear dynamics of interacting populations. World Scientific, 1998. 